\title{
Topology Mapping of Whole Pancreatic Beta Cells upon Drug Stimulation
}

\section{Kate White}

\author{
University of Southern California, Los Angeles, California, United States
}

To understand intracellular systems in biology, we must consider how organelles within a cell interact with one another and traffic in a crowded and chaotic environment that is in constant flux, similar to the importance of understanding traffic patterns in major cities. Advances in imaging technologies over the last decade have enabled the visualization of cell ultrastructure at increasing detail, opening the door to investigate cell structure and function relationships in more depth. Many high-resolution imaging methods require chemical fixing or sample slicing which alters the structural integrity of the cell and removes the cell from its native environment. To overcome these challenges, new approaches that maintain cellular structure are required. We applied soft X-ray tomography (SXT) to map the architectural changes that occur in pancreatic $\beta$-cells during insulin secretion. SXT is a label free imaging method that enables the investigation of the 3D architecture of whole cells and is ideal for investigating cell-to-cell structural variabilities. $1 \beta$-cells are secretory machines that produce and release insulin in response to glucose and are therefore are an excellent proof of principle target for cell mapping approaches. We used multiple stimuli to investigate the cellular organization changes that occur during insulin vesicle biogenesis, trafficking, and secretion. Additionally, we use machine learning methods for image analysis and autosegmentation to reduce manual human hours and provide a high throughput analysis pipeline. Furthermore, we can use the information from SXT along with live cell imaging to assemble a data-driven, dynamic model of organelles within pancreatic $\beta$-cells. The integration of data from different time and spatial scales is essential for characterizing the interplay between cellular structure and function during complex biological processes, which will revolutionize biological discovery, open doors to new dimensions of research, and accelerate advancements in healthcare.

\section{References}

1. Ekman, A.A., Chen, J.H., Guo, J., McDermott, G., Le Gros, M.A., and Larabell, C.A. (2017). Mesoscale imaging with cryo-light and X-rays: Larger than molecular machines, smaller than a cell. Biol Cell 109, 24-38. 